\title{
Contactless Mapping of Thoracic and Abdominal Motion: Applications for Seismocardiography
}

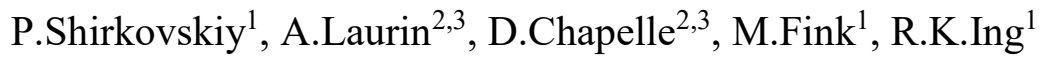 \\ ${ }^{1}$ Institut Langevin, CNRS, ESPCI Paris, PSL Research University, Paris, France \\ ${ }^{2}$ Inria, Palaiseau, France \\ ${ }^{3}$ LMS, Ecole Polytechnique, CNRS, Université Paris-Saclay
}

\begin{abstract}
Seismocardiography has been well studied in terms of analysis, applications, and methods of measurement. However, there remains a lack of research into the explanation and modelling of the involved phenomena. We propose a new contactless method to measure thoracic and sternal movements, demonstrate that it is adequate for typical seismocardiogram use.

An ultrasonic diagnostic tool called ICARE (CArdio REspiratory Imager) was designed to perform non-contact ultrasonic waves imaging on the thorax and abdomen. In addition to ICARE measurements an accelerometer was placed above the xiphoid of 3 participants (male, age $39 \pm 11$ ). Both ICARE and accelerometer measurements were performed concurrently.

Experimental results show the ability of the ICARE system to obtain $3 D$ seismocardiographic images with high frequency frame rate.

Furthermore, this technology could potentially be used to obtain cardio-vascular information in and out of clinical environments, significantly lowering the required time and effort.
\end{abstract}

Keywords - Ultrasonic imaging; surface motion; airborne; non-contact imaging; non-contact seismography; ultrasound vibrometry.

\section{Introduction}

Traditionally, seismocardiogram (SCG) and ballistocardiogram (BCG) systems use accelerometers to measure the vibrations produced by the heart contraction and blood ejection into the vascular tree. Alternatively, various techniques for non-contact heart and respiratory rate measurements are also proposed [1-3]. So far, these methods have mostly been confined to single point measurements on the thoracic wall.
Although some potential clinical uses of SCG and BCG have been proposed, its use remains largely limited to scientific laboratories, and few smartphone applications. This is in part due to the lack of physiological and physical understanding of the processes that underlie the phenomena.

To allow the study of the thoracic and abdominal systems as a whole, we have developed a system (ICARE, CArdio REspiratory Imager) capable of simultaneously detecting surface motion at any point of a region of interest. Because of cost and technical difficulty, the number of such methods is relatively small. We can cite acoustic holography, laser speckle imaging, and stereoscopic video [4-6].

While offering excellent accuracy and resolution, laserbased systems require a highly reflective surface and have the potential to cause serious harm. Acoustic holography has the disadvantage of being applicable only in nearfield, and the method becomes very tedious when trying to analyze low frequencies. Video methods are able to cover large areas but are severely limited in terms of time resolution and displacement sensitivity [6].

ICARE's use of acoustic waves has the advantage of being harmless, and presents a much lower cost of implementation than all other methods except the video methods. The use of such ultrasonic imaging to accurately detect obstacles/objects has been previously described [7]. The first patents for non-contact measurement of body movement with ultrasound were filed in the 70 s. More recently, a team described and validated a single channel, non-contacting ultrasound-based sensor for respiratory, non-respiratory, and caretaker movement of infants in an intensive care [8]. The technical capability for high frequency acoustical 3D surface vibrometry only starts to develop recently [9-10].

The goal of this study is to compare ICARE's capability of measuring SCG fiducial timings to the traditional accelerometer-based technique. A second goal is to perform qualitative examination of the $2 \mathrm{D}$ acceleration 
map under cardiac forces to develop hypotheses of the thoracic and abdominal systems as a whole.

The underlying aim was to explore the potential of ICARE to complement existing techniques in the performance meaningful medical measurements, and understand the mechanical principles behind cardiothoracic movements.

\section{Material and methods}

\subsection{ICARE device}

The 3D airborne non-contact ultrasound vibrometer is composed of three emission arrays arranged vertically (Figure 1 right) and one $16 \times 16$ square array of receivers (Knowles microphone FG-23629) in the middle.

Thirty-two transducers (Murata MA40S4S) are uniformly distributed over the area of each individual panel of $24 \times 24 \mathrm{~cm}^{2}$ dimension. The central panel combines both the radiating and receiving elements (256 microphone receivers and 32 emitting transducers) on the same plane. A camera is included in the experimental setup to overlay a visual representation of the participant to the acoustical images.

Because of a large difference between biological tissues and air impedances and because of the low roughness of the skin whose characteristic size is much smaller than the mean wavelength value $(8.6 \mathrm{~mm}$ at $40 \mathrm{kHz})$, the body surface acts as a specular surface. Ultrasonic waves are then principally reflected instead of being diffused.
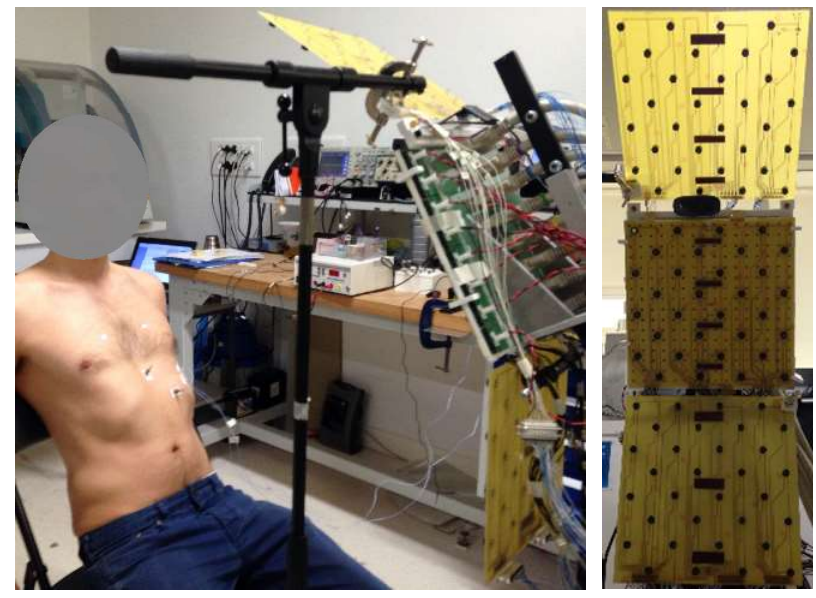

Figure 1. Left: description of the experiment. Accelerometer is placed on the sternum above the xiphoid and ICARE and accelerometer measurements are performed concurrently. Distance between the object and receivers is $72 \mathrm{~cm}$. Right: emission and receivers arrays. Central panel combines both the radiating and receiving elements.

The principal innovation of the ultrasonic imaging device is its ability to map surface motion of non-plane specular surfaces.

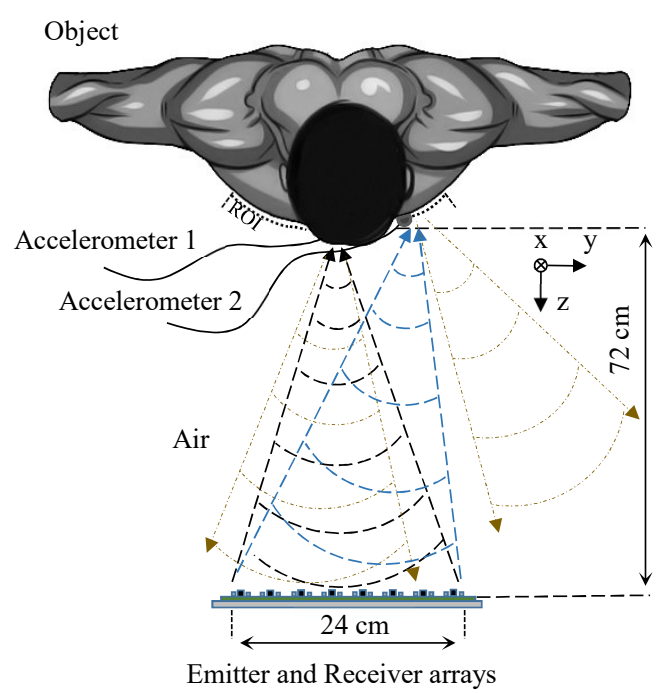

Figure 2. Sequential ultrasound emission during the measurement. Figure shows the non-reception of the waves by the receivers caused by non-plane surface of the body. ROI is the surface of the thorax and abdomen $24 \times 30 \mathrm{~cm}$.

To have a uniform spatial resolution over the whole imaged surface, each surface segment must reflect waves toward the entire receiver array. Therefore because of the specular behaviour of the body surface each surface segment must be illuminated with ultrasonic waves having a large wave-vector spectrum. Multiple panels are used to increase the surface of the emission aperture, and thus to reduce the non-reception of waves caused by a worth angulation (Figure 2). Data acquisition is performed on sitting participants (Figure 1).

\subsection{Signal processing}

A synthetic signal processing approach is used to ensure a high framerate imaging [11]. First the impulse response from emission channel $i$ to microphone receiver $j$ is acquired. Thereafter all the acoustic impulse responses are combined in post-processing to realize both emission and reception focusing. The ICARE system works in pulse echo mode.

Emitters are connected to a programmable D/A acquisition card with 32 channels (D-TACQ ACQ1001). Each of the 32 channels are connected to 3 transducers one for each emission panel. The emission waveform is a linearly modulated frequency signal that ranges from 35 $\mathrm{kHz}$ to $45 \mathrm{kHz}$. Its time duration is $320 \mu \mathrm{s}$. The signals are successively emitted by the $32 \times 3$ emitting transducers. Delay between two signal emissions is $160 \mu \mathrm{s}$. The duration of a sequence of emissions signals is $5.12 \mathrm{~ms}$ and, thus, the repetition rate is $195 \mathrm{~Hz}$.

Receivers are connected to a 256-channels A-D acquisition card (ACQ196, DAQ D-TACQ 16 bits, 400 
$\mathrm{kS} / \mathrm{s})$. Received signals are post-processed to achieve both emission and reception focusing. Finally, each imaged surface segment - pixel $2 \times 2 \mathrm{~mm}$ - is represented by a unique acoustic signal. Successive illuminations of the observed surface provide the several acoustic beamformed signals for each pixel of total surface of ROI. They are successively cross-correlated between each other to determine for each pixel the differential displacement, which correspond to displacement velocity in function of time. Acceleration is then computed by time differentiation.

The beamforming process provides $3 \mathrm{D}$ images $24 \times 30$ $\mathrm{cm}$ in dimension at a $195 \mathrm{~Hz}$ frame rate (thereafter the 3D movie of the surface velocity and acceleration). The pixel representing the sternum above the xiphoid is then identified and its acceleration time-series is used to compare with the accelerometer data acquired from the same location (Figure 3).

\subsection{Seismocardiograms}

An accelerometer is placed on the sternum above the xiphoid of all participants. Both ICARE and accelerometer (miniature piezotronic accelerometer, DJB Instruments, $1 \mathrm{mV} / \mathrm{g}, \quad 1 \mathrm{~Hz}-10 \mathrm{kHz})$ measurements are performed concurrently.

SCG signals from ICARE and accelerometer are both bandpass filtered at $15-60 \mathrm{~Hz}$ and the three chosen fiducial points are identified automatically using previously described software [12].

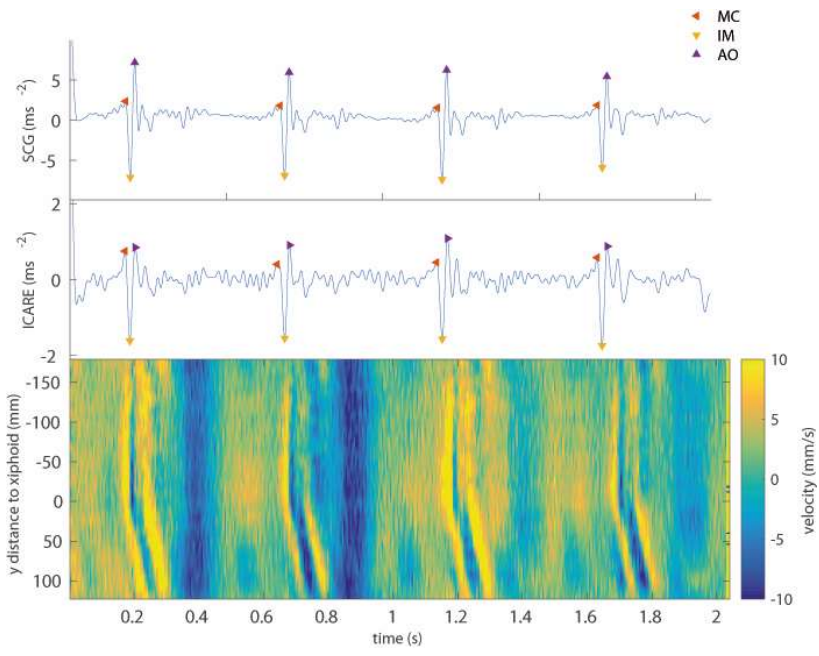

Figure 3. Time series for (Top) seismocardiogram acquired with an accelerometer, (Middle) seismocardiogram acquired with ICARE at the xiphoid, and (Bottom) the ICARE-measured velocity along the central vertical line for an example participant.

\section{Results}

The main goal of the study is to determine the capacity of the ICARE system to acquire valid SCG signals. When sampled at the point of placement of the accelerometer, the difference in timing of the first three SCG fiducial points was $-2.1 \pm 7.3 \mathrm{~ms}$ ("MC"), $3.9 \pm 7.3 \mathrm{~ms}$ ("IM"), and $-6.5 \pm 5.5$ ms ("AO") (Figure 3). Amplitude differences were 1.8 \pm 1.6 $\mathrm{m} / \mathrm{s} 2$ ("MC"), $1.5 \pm 3.2 \mathrm{~m} / \mathrm{s} 2$ ("IM"), and $1.9 \pm 2.6 \mathrm{~m} / \mathrm{s} 2$ (“AO”).


Figure 4. For an example participant, (Top) snapshots of the $2 D$ velocity map of the thoracic surface at times corresponding to "MC", "IM", and "AO" and (Bottom) a detail of the time series presented in Figure 3 Bottom. The data for each time point in Bottom represents the pixels at $x=0$ of the relevant snapshot analogous to Top.

A number of secondary observations are also performed on the resulting $2 \mathrm{D}$ time series. We estimate the velocity of the downward-propagating wave in the abdomen by computing the slope of the slanted sections (below the xiphoid) of each systolic wave train on the central-line velocity maps (Figure 3 bottom). The mean velocity for the 3 participants is $2.6 \mathrm{~m} / \mathrm{s}$. In contrast, the sections of the same velocity maps above the xiphoid are vertical.

It is also possible to observe the shape and position of the zone of maximal absolute velocity during the main fiducial points (Figure 4). For MC and IM timings, this zone is centred on the left-hand side of the participants' body and the bottom edge of it seems to coincide with the bottom edge of the thoracic cage.

\section{Conclusion}

The study presents preliminary results that support the capability of the ICARE system to perform SCG timing measurements. While the reporting of SCG amplitudes are much rarer in the literature, the ICARE system in its current configuration was not able to accurately measure 
them.

It is clear, though, that the real value of ICARE goes far beyond the measurement of SCG timings, by allowing the observation of the movements of the entire thorax and abdomen during heart beats.

The slanted sections of each systolic period, a wave propagation phenomenon below the xiphoid of $\sim 2.6 \mathrm{~m} / \mathrm{s}$ that is observed in all participants, is much too slow to be the pulse wave of the descending aorta, which travels at $\sim 10 \mathrm{~m} / \mathrm{s}$ in a healthy normotensive human [13]. This wave could potentially be caused by the heart pulling and pushing on the diaphragm, which then propagates in the general viscera.

This wave propagation also gains significance when seen in contrast with its absence in the sternal observations. These results are consistent with the hypothesis that the sternum moves as a solid object under heart forces, and would thus be unable to transmit the precise location of the source of minute cardiac events. These observations are also compatible with the idea that the thoracic cage reacts to cardiac forces as rigid whole, whose movements could be adequately represented with some relatively small set of vibration modes.

This study, while establishing the validity of ICARE in the most common use of SCG, i.e. the identification of a few fiducial points, has many limitations in terms of its broader scope. There is a severe trade-off in the system between time, velocity, and spatial resolutions which limits its ability to obtain precise SCG timings concurrently with precise localized acceleration measurements. The setup is constantly being improved to allow reasonable precision for all variables, and can be adapted to specific needs.

The small sample size of this study does not allow statistical generalization to entire populations. Future studies will incorporate many more participants and perform more robust quantitative analyses of the various phenomena observed on the 2D map.

As a valid method for measuring SCG fiducial timings, the ICARE system could provide a quicker, less invasive alternative to existing accelerometer-based techniques. In a routine visit to the doctor, for example, a device imbedded in the wall could perform measurements while the patient is standing on the weighing scale, minimizing effort and time.

ICARE has the potential to unlock a new dimension in the study of SCG, although more work is required to establish it firmly.

\section{Acknowledgements}

This work was supported by Chaire Safran - Fonds ESPCI Georges Charpak and European Research Council under the European Union's Seventh Framework Programme (FP7/2007-2013)/ ERC gran agreement $\mathrm{n}^{\circ} 610110$ (Helmholtz). We acknowledge the support of NVIDIA
Corporation with the donation of the Titan Xp GPU used for this research.

Alexandre Laurin would like to acknowledge the key role of Philippe Moireau and Sébastien Imperiale in the development the ideas concerning thoracic mechanics.

\section{References}

[1] Jure Kranjec, Samo Beguš, Janko Drnovšek, and Gregor Geršak. Novel Methods for Noncontact Heart Rate Measurement: A Feasibility Study. IEEE transactions on instrumentation and measurement, vol. 63, no. 4, April 2014, pp 838-847.

[2] A. D. Droitcour, O. Boric-Lubecke, and G. T. A. Kovacs. Signal-to-noise ratio in Doppler radar system for heart and respiratory rate measurements. Microw. Theory Tech. IEEE Trans., vol. 57, no. 10, pp. 2498-2507, 2009.

[3] S. D. Min, J. K. Kim, H. S. Shin, Y. H. Yun, C. K. Lee, and M. Lee. Noncontact respiration rate measurement system using an ultrasonic proximity sensor. Sensors Journal, IEEE, vol. 10, no. 11, pp. 1732-1739, 2010.

[4] G. Ramachandran and M. Singh. Cardiac Functional Analysis by Laser Speckle Interferometry, vol. 11, pp. 233239, 1989.

[5] A. Orimoto, H. Haneishi, N. Kawata, and K. Tatsumi. Monitoring and analysis of body surface motion caused by respiration, Ieice, no. October, pp. 523-526, 2009.

[6] M.-Z. Poh, D. J. McDuff, and R. W. Picard. Non-contact, automated cardiac pulse measurements using video imaging and blind source separation. Opt. Express, vol. 18, no. 10, pp. 10762-74, May 2010.

[7] J. Steckel. Sonar System Combining an Emitter Array With a Sparse Receiver Array for Air-Coupled Applications. IEEE Sens. J., vol. 15, no. 6, pp. 3446-3452, 2015.

[8] Gregory P. Heldt, and Raymond J. Ward, Evaluation of Ultrasound-Based Sensor to Monitor Respiratory and Nonrespiratory Movement and Timing in Infants. IEEE transactions on biomedical engineering, vol. 63, no. 3, 2016, pp 619-629.

[9] N.Jeger, M.Fink, R.K. Ing. Non-contact ultrasound stethoscope. J.Acoust. Soc. Am. 136, 2282, 2014.

[10] N. Jeger, M. Fink, R.K. Ing. 3D airborne ultrasound vibrometer for the detection of skin surface heterogeneities. IEEE International Ultrasonics Symposium, Tours, 2016.

[11] J. A. Jensen, S. I. Nikolov, K. L. Gammelmark, and M. H. Pedersen. Synthetic aperture ultrasound imaging. Ultrasonics, vol. 44 Suppl 1, pp.e5-e15, 2006.

[12] Laurin, Alexandre, et al. "Accurate and consistent automatic seismocardiogram annotation without concurrent ECG." Physiological measurement 37.9 (2016): 1588.

[13] Mancia, Giuseppe, et al. "2007 Guidelines for the management of arterial hypertension: The Task Force for the Management of Arterial Hypertension of the European Society of Hypertension (ESH) and of the European Society of Cardiology (ESC)." European heart journal 28.12 (2007): $1462-1536$

Address for correspondence.

Ros Kiri Ing

1 rue Jussieu, 75238 Paris Cedex 05, France

ros-kiri.ing@espci.fr 